\title{
OSNR aware Composition of Open and Disaggregated Optical Node and Network
}

\author{
Heng Liu, Adaranijo Peters, Miquel Garrich, Member, IEEE \\ and Georgios Zervas, Member, IEEE
}

\begin{abstract}
Function Programmable Optical Network has been recently proposed to enhance flexibility on optical transport based on Architectureon-Demand (AoD). The flexible synthesis of optical node architectures provided by AoD enables an open and disaggregated optical layer thanks to the available deep programmability. However, previous studies focus on how to synthesize a single node out of switching function blocks neglecting the optical signal-to-noise ratio (OSNR) impact, power imbalance effects due to diverse set of devices traversed per input-output configuration and network-wide implications. In this work, we present Optical Network-wide Function Synthesis (ONetFuS), an algorithm to compose AoD nodes that considers placement and configuration of both switches and amplifiers. ONeFuS minimizes OSNR degradation and deviation across channels and offers enhanced power balance performance. Moreover, ONetFuS addresses multiple-node scenarios to investigate cascading, transmission distance, and networking effects. We compare the number of optical crossconnections computed by our proposal against solutions in the literature. Results in network scenarios including number of components, power balance, OSNR variations and OSNR penalty reductions, prove the suitability of our proposed ONetFuS for Open and Function Programmable Optical Networks.
\end{abstract}

Index Terms - Architecture-on-Demand; Function synthesis and placement; Optical fiber networks.

\section{INTRODUCTION}

$\mathbf{T}$ Traditional optical backbone networks are characterized by fixed Reconfigurable Optical Add/Drop Multiplexers (ROADM) structures with hard-wired interconnection of

Manuscript submitted September 26th 2016.

H. Liu is with University of Bristol, Bristol BS8 1TH U.K.

A. Peters is with the University of Bristol, Bristol BS8 1TH U.K.

M. Garrich is with Dipartimento di Elettronica e Telecomunicazioni, Politecnico di Torino, Torino, Italy, and with CPqD - Centro de Pesquisa e Desenvolvimento em Telecomunicacoes, Campinas, Brazil.

Georgios Zervas is with Optical Networks group at the University College London, UK. devices [1]. These fixed ROADM nodes are difficult to reconstruct after projecting node architectures and realizing the deployment. Indeed, notable operational expenditure (OPEX) is required in such deployed traditional networks in case field operations are needed for upgrades due to new technologies (e.g., flex-grid, space division multiplexing SDM). A clear example is the current trend on Elastic Optical Networking [2], which is expected to provide benefits exploiting additional spectrum resources in already deployed fiber [3] but may require high long-term investments [4] or gradual migration of components [5]. Moreover, Wavelength Selective Switch (WSS)-based filtering effects in ROADMs may limit optical reach [6] notably at high bit-rates [7].

To overcome these challenges, the concept of Function Programmable Optical Network relying on Architecture on Demand (AoD) has been recently introduced in-line with industrial initiatives that propose open and disaggregated optical networks (e.g., OpenROADM [8]). In an Open AoDbased Function Programmable and Disaggregated Optical Network, the architecture of the nodes can be re-constructed to support different input requests [9]. All the components in each node are attached to the optical backplane arbitrarily and there are no fixed fiber connections between the components. Furthermore, the node can build different architectures according to the requests sent to the node by means of cross-connections in the optical backplane. From an industrial perspective, vendors recently followed the fundamental principle of module per function of the AoD approach [14] launching an open and disaggregated ROADM. As pointed out in [14], an AoD approach has the following benefits: a) reduced capital costs by using exactly the functionality needed, b) reduced operational cost by having lower footprint and lower power consumption c) enhanced flexibility that aids future-proofing the infrastructure and maximizing investment protection.

From an academic viewpoint, studies report that AoDbased Function Programmable Optical Networks provides more flexibility [10], scalability [11], availability [12], and resource and cost savings [13] than traditional optical networks. In more detail, recent studies target specific parameters to propose design mechanisms [10]-[12]. Precisely, a synthesis algorithm has been recently proposed to compose stand-alone AoD instances using Spectrum Selective Switches (SSSs), de-multiplexers (DEMUXs) and 
couplers [15]. However, this work presents several limitations because the use of MUX/DEMUX and couplers introduce a large number of optical cross-connections and cannot provide power control and power balancing capabilities of the traversing light paths. Besides, up-to-date analyses of functional programmable optical networks commonly rely on a set of assumptions and simplifications to investigate particular metrics such as cost [13] and resiliency [16], or to propose resource allocation policies in SDM networks [17]. However, these works lack of a detailed physical layer modeling and analysis avoiding the optical signal-to-noise ratio (OSNR) impact or power imbalance implications in the AoD design. Finally, only two recent studies, to the best of the authors' knowledge, address functional programmable optical nodes and networks with a detailed physical layer modeling and analysis. On the one hand, [18] investigates Erbium-doped fiber amplifier (EDFA) placement for channel power balance of a standalone function programmable optical node but neglecting OSNR and power imbalance effects in network-wide scenarios. On the other hand, [19] studies OSNR-aware, modulation format and transmission rate adaptive techniques to improve capacity or spectrum usage without providing EDFA placement nor power balance details.

In this context, synthesis algorithms for open and programmable nodes must be able to place SSS and EDFA on a single and multiple cascaded nodes to provide gain and output-power control of light paths. Moreover, it is essential to configure signal attenuation at each SSS in a per-channel basis to optimize OSNR performance and deliver minimum OSNR degradation and deviation across channels on different ports and light paths. Indeed, DEMUX and couplers used in [18] need to be replaced by SSSs to allow cross-connection reuse for multiple wavelength channels that have the same destination port. Furthermore, the SSSs can introduce granular power attenuation to each channel in the path besides the initial power loss of the components.

In this paper, we account for the referred challenges with the following contributions. First, we propose Optical Network-wide Function Synthesis (ONetFuS), an algorithm for function optical network programmability through placement and configuration of switching and amplification function blocks for a single and a cascaded set of AoD nodes. Second, we analyze its performance in terms of number of optical cross connections, OSNR variations, OSNR penalties and number of components used for different transmission requests in the AOD node. Third, we consider Quality of Transmission (QoT) and networking effects on a hop-by-hop Function Programmable Optical Network based on a set of cascaded AoD nodes.

The rest of the paper is structured as follows. Section II reviews related work. Section III presents the assumptions considered in this work and introduces ONetFuS with its application for a single and multiple cascaded AoD nodes. Section IV reports the simulation results and the performance in terms of OSNR and power balance is discussed. Section V concludes the paper.

\section{Literature REVIEW}

In this section, we review the latest literature of AoD- based open and disaggregated functional programmable optical nodes and networks.

\section{A. Open and Disaggregated Optical Networks}

Current trends on Elastic Optical Networking propose the use of optical spectrum resources in a flexible manner to increase capacity and network efficiency [2]. For instance, the Spanish nationwide optical backbone network can postpone new fiber deployment from 2020 to 2024 if EON is employed [3]. However, the migration from classical fixedgrid spectrum allocation towards flexible EON commonly requires notable long-term investments [4] or gradual migration of the WSS equipment [5]. Moreover, recent studies report the operational challenges of managing multi-vendor optical equipment with the need to adapt several interfaces just to perform an experimental end-toend resource provisioning [20]. Indeed, these operational challenges may hinder the potential of next-generation flexible-rate transponders [21].

In this context, academic initiatives to control optical components propose open YANG models [22] for EON [23], including monitoring functionalities [24] and management of sliceable transponders [25]. On the industrial side, the recent OpenROADM standardization initiative [8], proposes an interface for multi-vendor ROADM access and configuration. More specifically, OpenROADM targets the disaggregation (opening up) of traditionally proprietary ROADM systems and the Software Defined networking (SDN)-enablement of traditionally fixed ROADMs. Relevant in the industry, vendors are extending the open and disaggregation of ROADMs to implement the AoD principle [14]. As pointed out in [14], a module-per-function approach reduces CAPEX, reduces OPEX and offers an enhanced flexibility which future-proofs the infrastructure and maximizes the protection of the investment.

\section{B. Stand-alone Function Programmable Node}

The concept of function programmable optical node based on AoD was introduced in 2011 [9], precisely as a standalone node proposal. Subsequently, the first AoD synthesis algorithm was proposed in 2012 [11] to address its challenging configuration flexibility while reporting its scalability in terms of backplane cross-connections and building modules. As reported in [11], synthesis algorithm commonly refers to the technique that performs the architecture computation and composition. Precisely, a reduction of synthesized modules in AoD nodes targets an increase of the optical transparent reach overcoming WSS filtering impairments [6], [7]. Further characteristics of AoD have been analyzed in the literature following a stand-alone approach, including: its availability leveraging on the reliability of building modules and optical backplanes [12], the flexibility of the complete node [10], and its power consumption [26].

A recent and comprehensive study of AoD includes a synthesis algorithm [15] that improves the original work reported in [11] by checking for fiber switch requests, waveband (i.e. super-channel composed of multiple optical carriers) switch requests and wavelength switch requests separately. Then, different types of components for optical node design, including SSSs, DEMUXs and couplers are 


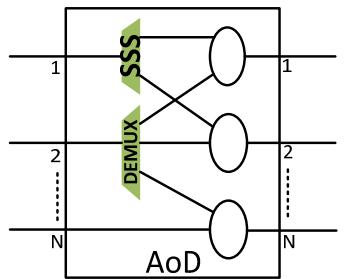

(a) (b)

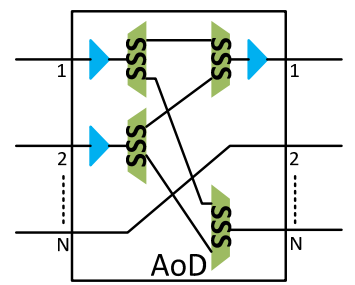

Fig. 1. AoD node instances computed by (a) the algorithms in [11], [13], [15], and (b) the algorithm proposed in this work.

placed according to the different requests. Furthermore, the synthesis algorithm in [15] considers sub-wavelength switching requests and piezoelectric lead lanthanum zirconate titanate (PLZT) switches are placed to account for this type of traffic needs. Note that [15] also includes a comprehensive cost analysis to report the economic benefits of AoD against traditional ROADM architectures. In particular, [15] shows the convenience of AoD against ROADMs for the fixed- and flexible-grid spectrum allocation scenarios, and even when TDM subwavelength traffic is considered. In terms of architectural modelling, the work in [11] uses a two-stage configuration, whereas [15] considers a three-stage AoD configuration to place PLZT switches at the second stage (middle stage) while the first and the third stages are used for SSSs, DEMUXs and couplers.

However, these works present several limitations because the use of MUX/DEMUX and couplers introduce a large number of optical cross-connections as one cross-connection per wavelength and per fiber is required to enable frequency switching. Moreover, MUXs/DEMUXs cannot attenuate the power of the traversing light paths limiting power control and balancing capabilities at each port of the node.

\section{Function Programmable Optical Networks}

Leveraging on AoD nodes, functional programmable optical networks have been recently proposed. Related work includes an Integer Linear Programming (ILP) formulation and a heuristic for a scalable, effective, and cost-efficient network design [13]; a network-wide resiliency analysis using AoD nodes [16]; a dynamic connection provisioning strategy which exploits AoD idle redundant modules to reduce network blocking [27]; an AoD-based network provisioning scheme accounting on traffic dynamicity [28]; a power-consumption comparison between AoD and ROADM nodes in network scenarios [29]; and SDM networks with AoD-based optical nodes [17]. It is worthwhile to mention that these works report the usefulness of AoD nodes to support both static [16] [19] [29] and dynamic [13] [17] [27] [28] requests. For instance, [16] reports benefits when deploying redundant AoD nodes over doing so in hard-wired ROADMs in terms of operator revenues, mean down times reduction, and redundancy costs reduction. Moreover, note that the economic viability of AoD in a network scenario considering cost aspects is widely analyzed in [13]. In particular, [13] shows that, even at high loads, a network with AoD nodes achieves savings of switching modules up to $40 \%$ compared to static ROADMs through a proper network design. Furthermore, [13] proves that by diminishing the spectrum selective switches the overall power consumption of the network decreases by more than $25 \%$ for high loads. However, these works commonly consider a simplified optical network model accounting mainly for the number of SSS modules and/or their characteristics (e.g., port number, power consumption or dependability) neglecting amplification and other optical/physical-layer aspects.

In summary, [11] and [15] for stand-alone and [13] for network-wide are precursors that created AoD synthesis algorithms addressing many unprecedented challenges. However, power balance and OSNR constraints of the node were not considered and they are crucial for reliable optical network operations.

\section{Power Balance and OSNR}

In an AoD node, the architecture and function placement is re-configured to account for different requests. Therefore, signals exiting at the same port may experience different power loss from the use of diverse types and number of function modules. As such, it is essential to guarantee power balance when synthesizing the AoD node in order to provide uniform and optimum end-to-end OSNR and bit error rate (BER) performance.

It is worthwhile to mention two related works. On the one hand, H. Yuan et al. proposed and compared in [18] three predefined static methods for the EDFA placement in standalone AoD nodes. Specifically, SSSs replace couplers in the architecture to enable spectrum switching and EDFAs are placed account for optical amplification. The combination of SSSs and EDFAs offer the possibility to balance the optical power of the signals. In the first two methods, signals are amplified and balanced before coupling at the SSS in the last stage. In the third method, EDFAs are placed after the last switching stage of different ports. The power balance is achieved by the flexible-grid attenuation capability of the SSSs. From the OSNR performance perspective, [18] concludes that the third method performs significantly better than the former two. On the other hand, [19] proposed OSNR-adaptive modulation format and symbol rate transmission techniques to improve capacity or spectral efficiency. However, [19] does not address EDFA placement nor power balance equalization employing SSSs.

In this work, we address this challenge proposing OSNR and Power Balance aware EDFA Placement (OPB-EDFAPlacement), an algorithm to consider EDFA placement, its configuration in terms of output power and fine gain attenuation per wavelength performed at each SSS. OPBEDFA-Placement is the second major step within ONetFuS and is analyzed for both single node and multiple cascaded node composition to evaluate signal performance on a multihop network environment. Illustratively, Fig. 1(a) and 1(b) presents different components used to compute AoD node architecture in previous works (e.g., [11], [13], and [15][14]) and in this work respectively.

\section{Synthesis OF AoD NodE AND NETWORK}

Figure 2 shows the Function Programmable Optical Network, which consists of a central controller and a local controller for each AoD node. Requests that contain the information of all the source-destination pairs and wavelength channels are received in the central controller as network traffic matrices. The central controller processes 


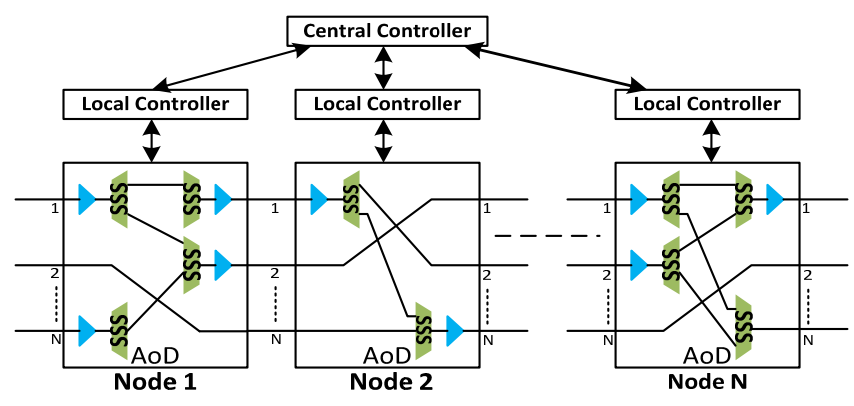

Fig. 2. Composition of the Network

each given network traffic matrix computing end-to-end routes and preforming the wavelength assignment, i.e. as in classical path computation element (PCE) engines ${ }^{1}$. Subsequently, the central controller executes the synthesis algorithm (explained below) to identify the network functions and configurations required on every AoD node according to each end-to-end request, which is now expressed as route and wavelength. This process in the central controller is performed following a hop-by-hop approach. Once the architectures are computed, commands for node construction are sent to the local controller of each AoD node along the end-to-end path. Finally, the local controller performs low-level instructions according to the northbound interface supported (i.e. TL1, NETCONF, REST, OpenFlow). The network is considered to be composed when all the nodes are synthesized and the devices are configured.

Figure 3 presents the Optical Network-wide Function Synthesis (ONetFuS) algorithm. ONetFuS is an improved synthesis algorithm compared to previous proposals (e.g., [11], [13], and [15]) because the identification of the network functions, configurations, architectures computation and composition now includes OSNR and power balance operations in a network-wide perspective. ONetFus is divided in two steps. Crucially, it is the first study exploring the OSNR penalty of the AoD architecture against standard ROADM architctures. The first step executes the Switching Synthesis and Placement Algorithm (SSPA) which first places and configures the SSSs for multiplexing/demultiplexing functions and to enable power equalization of the signals. Then, the SSPA sets the required optical cross-connection to provide the desired functions and in-out interconnections. The second step runs the OSNR and Power Balance Aware EDFA Placement (OPB-EDFA-Placement) algorithm that places and configures the amplifiers, performs the power balance (channels' equalization) and monitors the OSNR.

\section{A. Switching Synthesis and Placement Algorithm}

The Switching Synthesis and Placement Algorithm (SSPA) addresses the AoD synthesis challenge using a novel approach based on a two-stage logical architecture of SSSs and optical cross-connection reuse. On the one hand, SSPA considers all kinds of switching requests in WDM networks, including fiber, waveband and wavelength switch requests.

\footnotetext{
${ }^{1}$ In this work, we analyze a cascaded setup of AoD nodes to focus on OSNR and power balance effects. The impact of different routing algorithms (i.e., path computation) in diverse network topologies on the number of devices, OSNR and power balance effects is left for future work.
}

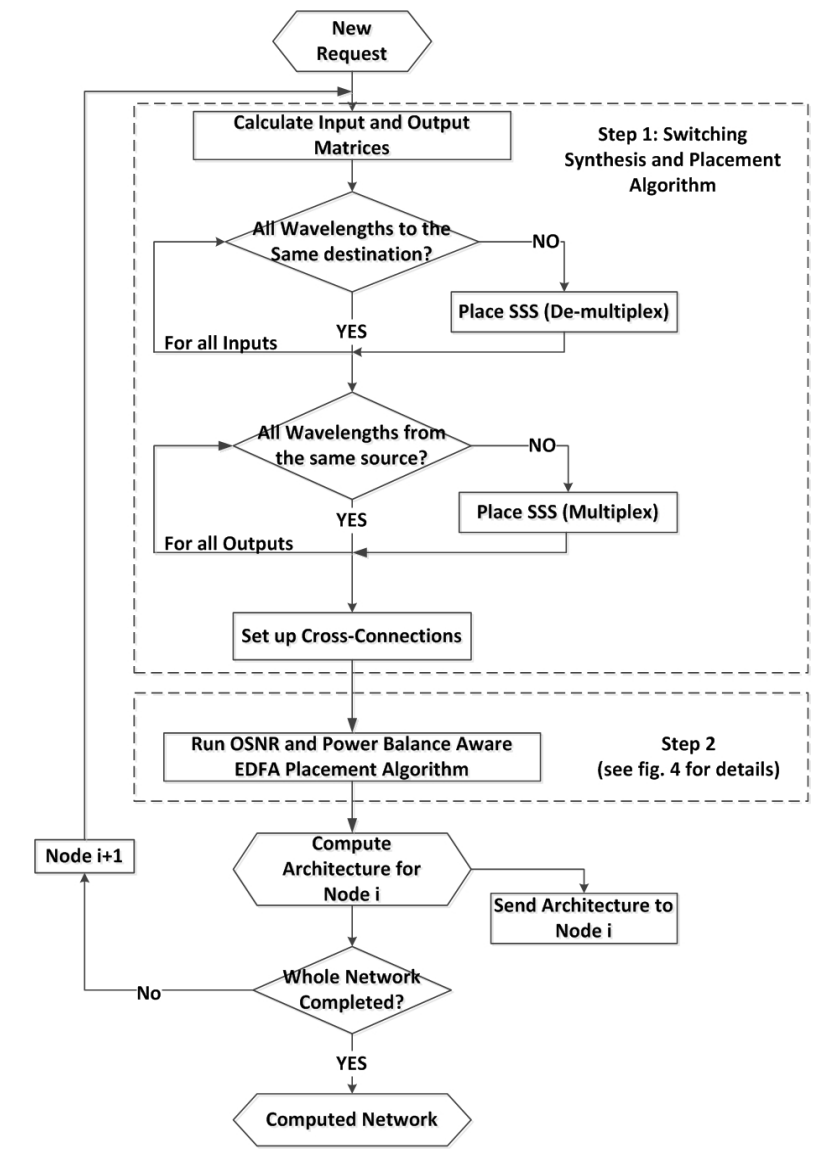

Fig. 3. Optical Network-wide Function Synthesis (ONetFuS) algorithm, which executes two steps at each node: first, the Switching Synthesis and Placement Algorithm (SSPA), and second the OSNR and Power Balance aware EDFA Placement (OPBEDFA-Placement) Algorithm.

DEMUXs and couplers used in [11] and [15] are replaced by SSS for flexible multiplexing and de-multiplexing. Critically, this algorithm is able to consider the OSNR as part of this placement and configuration policy. Signals passing through SSS can be attenuated at individual basis [30] besides the minimum insertion loss. Moreover, SSS can mitigate filtering effects of WSS [6]. It is important to note that the decision for placement of the EDFA and the OSNR performance depends on the node architecture computed by the SSPA. Unlike the AoD synthesis algorithm reported in [15], sub-wavelength switching is not considered in this work.

With the use of SSS, wavelength and waveband requests are able to reuse the same optical cross-connection when they share source or destination ports. This enables a reduction of the number of optical cross-connections and permits the replacement of the three steps detection used in [11] by correlating the requests (matrices) with the input/output ports.

The sequence of actions executed in the SSPA is shown in step 1 of Fig. 3. Given a new request that comprises wavelength and wavebands channels with their destination information, the central controller starts by computing the first AoD node. The input and output matrices for all the fibers in the AoD node are calculated considering the global network request. The matrices contain the information of all 


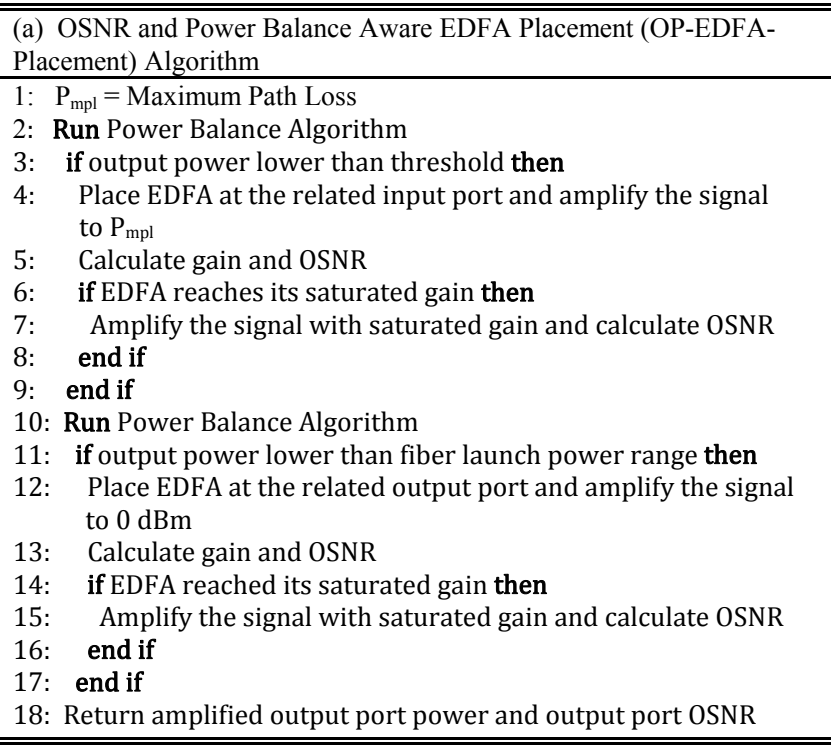

(b) Power Equalization Algorithm

1: for output port (i) $\in$ all output ports do

2: if there is second stage SSS (multiplex) then

3: for all channels passing through SSS do

4: $\quad$ Compute the maximum power can achieve in this path

5: end for

6: $\quad$ Select the minimum power among the paths and add attenuations to other paths to get power balance

7: $\quad$ Output Power $(\mathrm{i})=$ the minimum power among the paths

8: else

9: $\quad$ Output Power $(\mathrm{i})=$ the maximum power can be achieved

10: end if

11: end for

12: Return Output Power

Fig. 4. (a) OSNR and Power Balance Aware EDFA Placement Algorithm. (b) Power Balance Algorithm: Threshold = -10 dBm; Saturated gain $=25 \mathrm{~dB}$; Optical fiber launch power range $=-3 \mathrm{dBm}$ to $3 \mathrm{dBm}$.

the channels used in the input/output ports and the destination/source ports of those channels. The SSPA checks the destination ports for all the channels in each input port. In case that all the channels in the port have the same destination, there is no need for any spectral switching operation. Otherwise, a SSS is placed at the input port to de-multiplex the channels towards their different destination. Similarly, the SSS placement at the second stage of the AoD node depends on the output port matrices computed by the algorithm. For ports with different channel sources (incoming channels), a SSS is placed in order to combine them. After checking all input and output ports, optical cross-connections between the required SSSs and ports are set up. Then, the SSPA is completed and ONetFuS passes to the next step.

\section{B. OSNR and Power Balance aware EDFA Placement (OPB-EDFA-Placement) algorithm}

The second step shown in Fig. 3 is the OSNR and Power Balance Aware EDFA Placement (OPB-EDFA-Placement) algorithm. EDFAs can be placed either before separating the wavelength channels at the first stage and/or after combining them at the second stage. According to the noise figure performance of EDFA against different input power levels, very low input power levels (i.e. in the order of -25

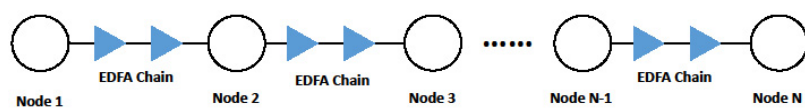

Fig. 5. Cascade of AoD nodes interconnected by EDFA Chain

$\mathrm{dBm})$ are not desirable. Therefore, and due to insertion loss of node function blocks, EDFAs are preferentially first placed at the input ports of the AoD node. Moreover, in order to minimize the number of EDFAs used in the node, the output power of the EDFA placed at the input port is assumed to be able to compensate for the maximum path loss. In particular, an EDFA at the input port compensates the loss of two SSSs (i.e. $10 \mathrm{~dB}$ plus additional signal attenuation required for power balance) and the loss of up to four cross-connections (i.e., $4 \mathrm{~dB}$ ) while achieving a $\sim 0 \mathrm{dBm}$ output power (fiber link launch power).

The process of the OPB-EDFA-Placement algorithm is shown in Fig. 4(a), including the Power equalization algorithm, which is shown in Fig. 4(b). The AoD node computes the output power level of all channels of each port based on the input power and input OPB-EDFA-Placement Algorithm. This algorithm balances the power of all wavelengths from all incoming ports directed to the same output port thus requiring a SSS at the second stage. The algorithm computes the attenuation required to equalize all the candidate paths to the minimum power level among them. Then, the SSS attenuates the channels accordingly in order to achieve the same power level when combining them at each desired output. The output power is detected and checked whether it is lower than a certain threshold, (i.e. $-10 \mathrm{dBm}$ in this work). Note that this threshold value depends on the noise figure of the EDFA and it can be modified in different situations. If the output port power is lower than the threshold, an EDFA is placed at the input port to amplify in order to recover all the component loss in the path and achieve a $0 \mathrm{dBm}$ at the output port. The gain of each EDFA is calculated and if the EDFA reaches its saturation regime, the maximum gain is used.

After placing the required EDFAs at the input ports, OSNR and the output power values are calculated based on the amplified input port power. If the output power does not meet the requirement for launching to an optical fiber, which is $-3 \mathrm{dBm}$ to $3 \mathrm{dBm}$ in this work, EDFAs are placed at the output ports. This situation may occur due to low input powers or EDFAs reaching their saturated gain at the first stage. Finally, the output power is controlled, the output OSNR is computed and the AoD composition is completed.

\section{Network-wide Composition}

The network fabricated in this work is a chain of cascaded AoD nodes as shown in Fig. 5. EDFAs are used for optical amplification in order to account for fiber loss between two consecutive nodes. Indeed, note that this EDFA chain in the fiber line is a major contributor to the noise accumulation compared to the fewer EDFAs synthesized at each AoD node, when required.

Figure 6 shows an example of the computation of an Open AoD-based Function Programmable and Disaggregated Optical Network using our proposed ONetFuS. Each 


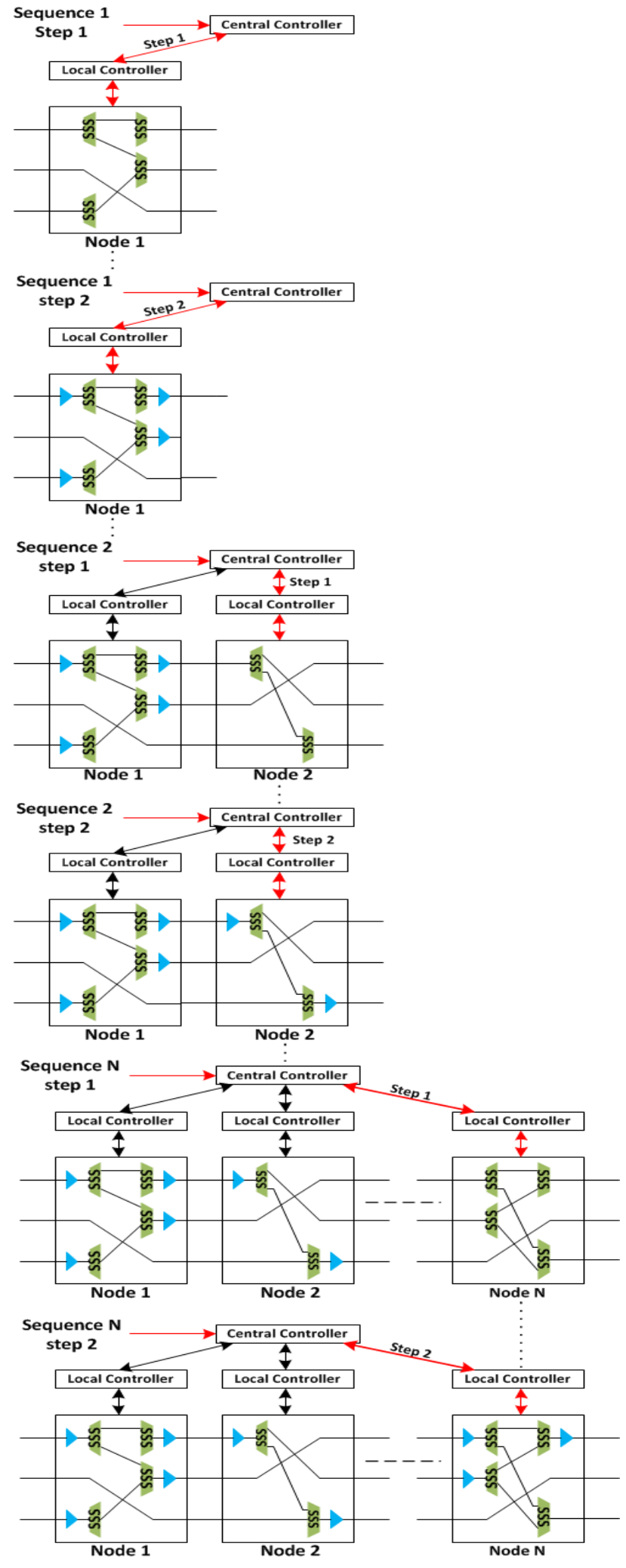

Fig. 6. Implementation of the Network

sequence corresponds to an ONetFuS operation on an AoD node. Each sequence is divided in two steps in order to execute SSPA and OPB-EDFA-Placement algorithms. Specifically, in step 1 of sequence 1 shown in Fig. 6, the first node of the network receives the matrix from the central controller while the rest of the nodes wait for their local
TABLE I

ASSUMPTIONS FOR SIMULATION

\begin{tabular}{lc}
\hline \hline \multicolumn{1}{c}{ Assumptions } & Value \\
\hline Cross-connection Loss & $1 \mathrm{~dB}$ \\
SSS Loss & $5 \mathrm{~dB}$ \\
SSS Minimum Attenuation & $0 \mathrm{~dB}$ \\
SSS Attenuation Granularity & $1 \mathrm{~dB}$ \\
EDFA Saturated Gain & $25 \mathrm{~dB}$ \\
Fiber Loss & $0.2 \mathrm{~dB} / \mathrm{km}$ \\
Node Chain EDFA Output Power & $0 \mathrm{~dB}$ \\
Distance Between Node Chain EDFA & $50 \mathrm{~km}$ \\
$n_{s p}$ is the population- inversion factor & 1 \\
Planck Constant $h$ & $6.63 \times 10^{-34} \mathrm{~J} \cdot \mathrm{s}$ \\
Optical Bandwidth & $100 \mathrm{GHz}$ \\
\hline \hline
\end{tabular}

information. The SSPA places SSSs and establishes the optical cross-connections in the first node according to the demand. Step 2 of sequence 1 in Fig. 6 shows process of OPB-EDFA-Placement algorithm where light path power equalization and EDFAs in the first AoD node are placed. After this two steps, the architecture of the first AoD node is completed, and the architectures of the following nodes are computed and built in a hop-by-hop process

\section{Results}

This section reports our simulation results. The analysis includes the optimization of the number of crossconnections, the OSNR performance, and the number of EDFAs used in one and multiple AoD nodes in a cascaded setup as well as the power balance performance. Although the route computation plays a remarkable role in the number of components to be used in the AoD nodes, in this work we focus on a cascaded nodes setup to analyze the OSNR and power balance effects regardless potential path alternatives. The study of different network topologies to account for path diversity is left for future work.

Table I lists the main assumptions considered for the simulations in this section. The proportion of requests of all the nodes in the network is assumed to be the same and the architectures are composed according to the requests. OSNR and OSNR Penalty (noise figure) presented in this section are calculated following the equations available in [31]:

$$
\begin{gathered}
O S N R=\frac{S}{N}=\frac{\text { averagesignal power }}{\text { total noise power }} \\
\text { noise figure }=\frac{O S N R_{\text {in }}}{O S N R_{\text {out }}}
\end{gathered}
$$

The noise power introduced by the Amplified Spontaneous Emission (ASE) effect is computed as

$$
P_{A S E}=2 h v n_{s p}(G-1) B
$$

where $n_{s p}$ is the population-inversion factor, $h$ is the Planck constant, $B$ is the optical bandwidth, $G$ is the gain of the amplifier and $v$ is the optical signal frequency.

\section{A. Scalability Analysis}

Here we analyze the number of optical cross-connections used in the AoD node in response to different demands and for different number of ports. We compare the optical crossconnection required between the AoD Synthesis Algorithm in [11] and the SSPA in this work. By replacing the MUXs/DEMUXs (AWGs) and couplers with SSSs, the 
TABLE II

PARAMETERs For STAND-ALONE AoD Node SimUlation

\begin{tabular}{ccc}
\hline \hline Parameters & (a) & (b) \\
\hline Port Number (N) & $\begin{array}{c}5 \text { to } 25 \\
\text { (interval 5) }\end{array}$ & 5 to 25 (interval 5) \\
\hline Channels (W) & 60 & 60 \\
\hline Port Load (P) & $\begin{array}{c}0.05 \text { to 0.95 } \\
\text { (interval 0.1) }\end{array}$ & 0.95 \\
\hline Fiber Switch Requests (F) & 0 & 0 to 0.9 (interval 0.1) \\
\hline \hline
\end{tabular}

Results are average of 500 simulations for each set of parameters

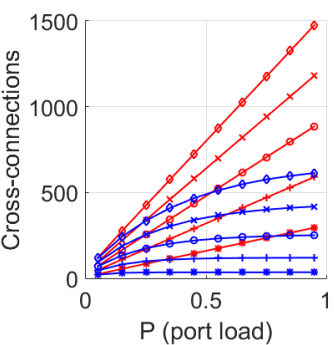

(a)

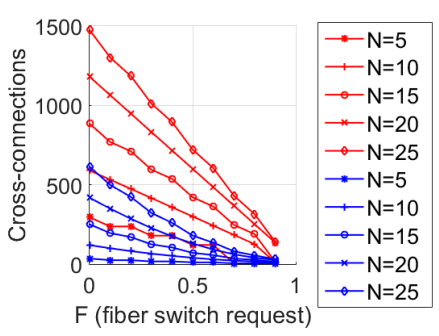

(b)
Fig. 7. Number of Cross-connections Used

(a) $\mathrm{P}=0.05 \sim 0.95, \mathrm{~F}=0$; (b) $\mathrm{P}=0.95 ; \mathrm{F}=0 \sim 0.9$

Red - AoD Synthesis Algorithm in [11]. Blue - Our proposed SSPA

optical cross-connections can be reused significantly. Table II presents the parameters used in this particular simulation. Port load $(\mathrm{P})$ represents the proportion of active wavelength channels in each port. This proportion is maintained for all the ports, i.e., the load is uniformly distributed. Fiber switch request (F) represents the proportion of the fiber switching in the simulation to account for channels of a given input are all switched to the same output. Each set of parameters (N, P, F) characterizes a fixed set of requests for a simulation run. The results presented here are obtained averaging the outcome of 500 simulations for each set of parameters.

In Fig. 7(a) we observe that as the port load increases, the number of optical cross-connections does not increase linearly using SSPA as it does using the algorithm in [11]. This is due to the reuse of optical cross-connections. In particular, high port loads increase the possibility that optical cross-connections can be reused. Specifically, for an AoD node of 25 ports (i.e. $25 \mathrm{SMFs}$ for $\mathrm{N}$ directions/degree or three 7-core Multi-Core Fibre (MCF) with 3 add/drop cores) and a port load of 0.95 , the SSPA can reduce the total optical cross-connections by about $60 \%$. It shows that even commercial 3D-MEMS based fiber switches with 640 ports can accommodate the number of cross-connection required. Figure 7(b) shows the scenario in which the AoD node receives an increasing proportion of fiber switch requests. This scenario considers a network considerably loaded where paths can be re-allocated and re-optimized to exploit fiber switching. The trend is similar for the two algorithms because the number of cross-connections linearly reduces as the fiber switch requests increase.

\section{B. OSNR Variation against (Non-)Flat Input Power}

Here we analyze the OSNR variation against different input powers considering two main scenarios in a cascaded setup of AoD nodes. On the one hand, the flat input power scenario accounts for a perfect equalization of all the input powers at all the input ports in all the nodes of the network. On the other hand, a non-flat input power scenario
TABLE III

PARAMETERS For CASCADED AOD NODES SIMULATION

\begin{tabular}{lc}
\hline \hline \multicolumn{1}{c}{ Parameters } & Values \\
\hline Port Number (N) & 5 \\
Channels (W) & 60 \\
Port Load (P) & 0.5 \\
Fiber Switch Requests $(\mathrm{F})$ & 0.8 \\
Waveband Requests $(\rho)$ & 0 \\
Distance between Nodes & $100 \mathrm{~km}$ \\
Input OSNR & $30 \mathrm{~dB}$ \\
Non-Flat Input Power & {$[-10,-5,0,5,10] \mathrm{dBm}$} \\
Flat Input Power & {$[0,0,0,0,0] \mathrm{dBm}$} \\
\hline \hline
\end{tabular}

Results are average of 500 simulation runs

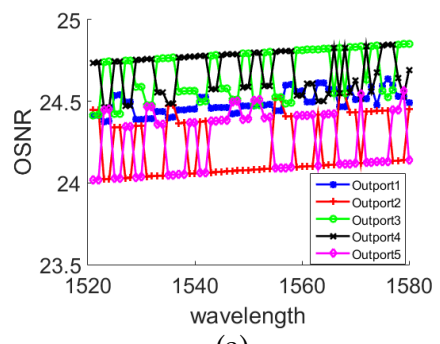

(a)

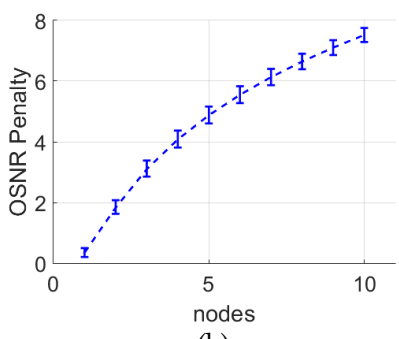

(b)
Fig. 8. OSNR Variation with Non-Flat Input Power. (a) OSNR at the five output ports of the sixth node, and (b) OSNR penalty against different hops.

represents a variation on the input power in different input ports to emulate, for instance, potential differences on transmission links. Table III shows the parameters for the simulations of these scenarios. The results reported here are obtained averaging 50 simulations for each set of parameters.

Figure 8 and 9 address the non-flat and flat input power scenarios, respectively. Figure 8(a) shows the OSNR measured at the five output ports of the sixth node in the network. We observe an output OSNR variation around 0.5 $\mathrm{dB}$ in the non-flat scenario. However, in Fig. 9(a), we observe a smaller variation of the output OSNR at the sixth node compared to Fig. 8(a) although the mean OSNR is the same. Figure 8(b) and 9(b) show the average OSNR penalty and the variation against different hops in the network, again, for the non-flat and flat input power scenarios, respectively. We observe that both scenarios follow the same trend in terms of mean OSNR regardless the number of traversed nodes. However, when all the input power of the ports are $0 \mathrm{dBm}$ (flat scenario), the OSNR variation is reduced to about $0.2 \mathrm{~dB}$, regardless the number of traversed nodes.

\section{OSNR Penalty against Input Power and Hops}

To evaluate in greater depth the impact of different input powers in a scenario with cascaded AoD nodes, we analyze the OSNR penalty against different input power and multiple hops. The distance between two adjacent nodes is assumed to be $100 \mathrm{~km}$. Results presented here are the average of 500 simulations for each set of parameters. From Fig. 10(a), we can see that when the input power is lower than $-10 \mathrm{dBm}$, the OSNR penalty increases exponentially at the first nodes. However, as the number of hops increases, the OSNR penalty increases linearly but with different proportions. In more detail, at the output port of the second node, the OSNR penalty diverges about $4.2 \mathrm{~dB}$ between the input power of $-20 \mathrm{dBm}$ and $10 \mathrm{dBm}$. But at the output port of the tenth node after transmission, the difference between 


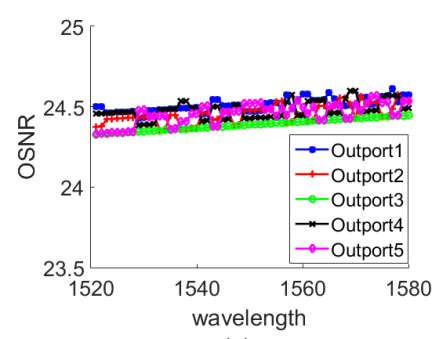

(a)

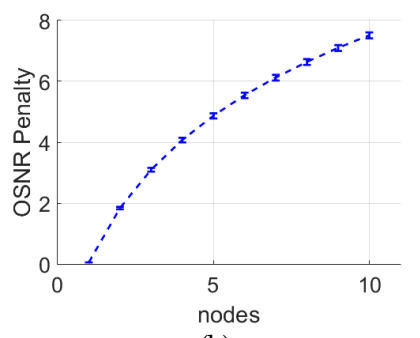

(b)
Fig. 9. OSNR Variation with Flat Input Power (a) at the five output ports of the sixth node and (b) penalty at different hops.

these two scenarios is reduced to about $1 \mathrm{~dB}$. This is because of a greater effect on the OSNR penalty when the input powers are low. However, for the cases with high input power, the number of hops in the transmission path affect in a higher proportion the OSNR penalty.

In Fig. 10 (b), we analyze the OSNR penalty against a small range of the input power levels, which is from $-5 \mathrm{dBm}$ to $5 \mathrm{dBm}$. We observe that the OSNR penalty performs slightly different between $-2 \mathrm{dBm}$ to $2 \mathrm{dBm}$. Generally, the OSNR penalty decreases as the input power level increases, except for the transitions from $-3 \mathrm{dBm}$ to $-2 \mathrm{dBm}$ and from $2 \mathrm{dBm}$ to $3 \mathrm{dBm}$ in which the OSNR penalty increases as the input power increases. This is caused by the threshold and the optical fiber launch power range set in the OPBEDFA-Placement algorithm. In particular, an EDFA is placed at the output port when these specific power-budget transitions occur. Indeed, more noise is introduced into the system due to this additional amplifier.

\section{OSNR Penalty against Node Distance and Hops}

The distance between the two adjacent nodes in the network also affects the OSNR performance. Longer distances between adjacent nodes require a high number of EDFAs for light path establishment. We assume that EDFAs are placed at each $50 \mathrm{~km}$. Figure 11 shows the OSNR penalty against adjacent node distance and number of hops (nodes). We observe that the OSNR penalty increases linearly as the distance increases for values higher than $250 \mathrm{~km}$ of adjacent node distance. For scenarios with a distance shorter than $250 \mathrm{~km}$, the OSNR penalty for an extra $50 \mathrm{~km}$ is slightly higher. During the transmission, optical signals suffer from the fiber attenuation and EDFAs amplify the signal to $0 \mathrm{dBm}$ with an automatically calculated output power for every $50 \mathrm{~km}$. Therefore, after several EDFAs, the signal power of different ports becomes similar, and the OSNR penalty trends to be linear as the distance increases.

Figure 11 also shows the OSNR penalty against different hops with different adjacent nodes distances. Longer distances between the adjacent nodes definitely introduce more noise to the signal. However, as the number of hops increases, the OSNR penalty between adjacent hops decreases. From Fig. 11 we can also see that the OSNR penalty from node 9 to node 10 is similar regardless the distance between two adjacent nodes.

Note that the OSNR penalty is mainly related to the distance and hops between the starting node and destination node. Therefore, still in Fig. 11, the minimum OSNR penalty is $1.83 \mathrm{~dB}$ when there is only one hop of 100 $\mathrm{km}$ link distance. On the opposite case, at the tenth node port of the $600 \mathrm{~km}$ scenario, the OSNR penalty reaches up to $14.22 \mathrm{~dB}$. It is also interesting to observe that the OSNR

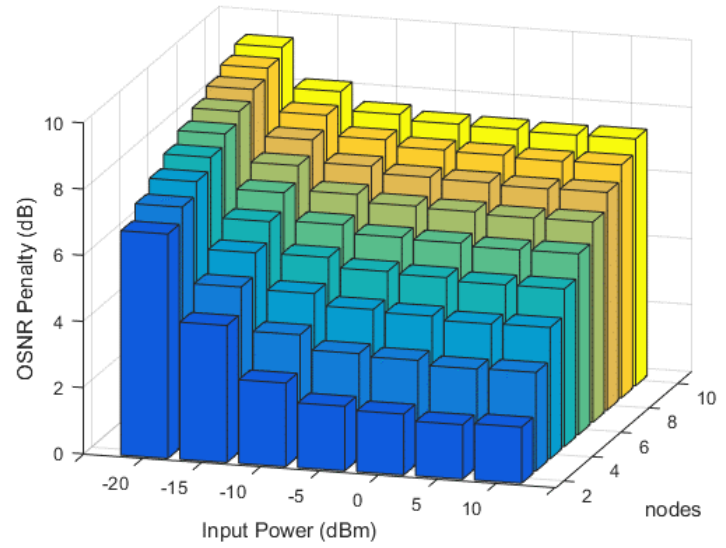

(a) Input Power $=-20 \mathrm{dBm}$ to $10 \mathrm{dBm}$ (interval $5 \mathrm{dBm}$ )

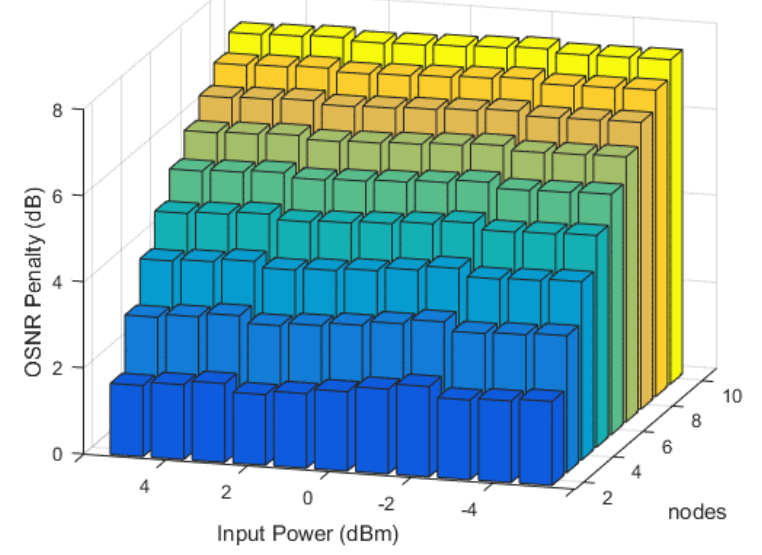

(b) Input Power $=-5 \mathrm{dBm}$ to $5 \mathrm{dBm}$ (interval $1 \mathrm{dBm}$ )

Fig. 10. OSNR Penalty against Input Power and Hops

penalty is $5.84 \mathrm{~dB}$ for a single hop with link distance equal to $600 \mathrm{~km}$, and the OSNR penalty experienced at the seventh node with $100 \mathrm{~km}$ link distance $(600 \mathrm{~km}$ between source and destination) is $6.11 \mathrm{~dB}$. Indeed, the option of the link with multiple hops has slightly higher OSNR penalty. However, being such difference of only $0.3 \mathrm{~dB}$, it is relevant to note that the impact of traversing six AoD nodes is almost transparent compared to a straight line with amplifiers. Therefore, AoD offers switching capabilities and function placement with a minimal impact on the OSNR performance. Moreover, these results obtained considering

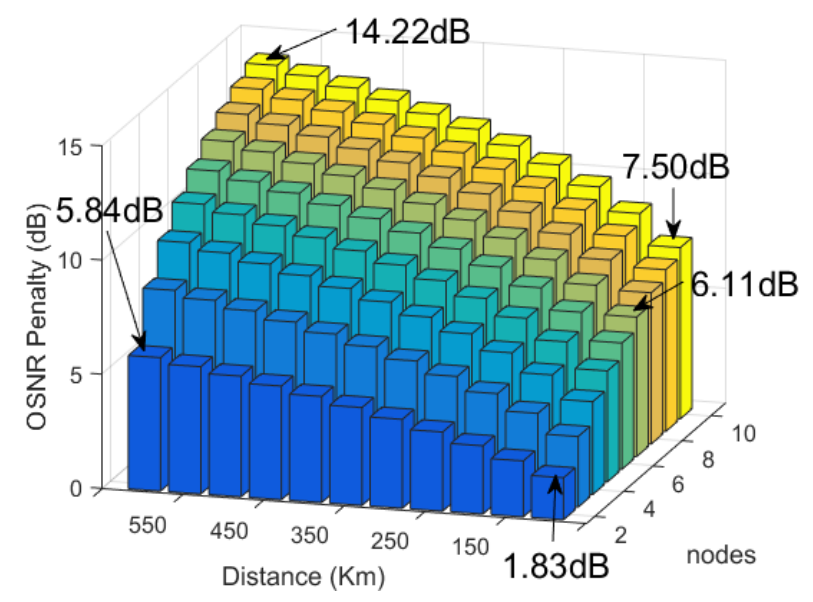

Fig. 11. OSNR Penalty against Node Distance and Hops 


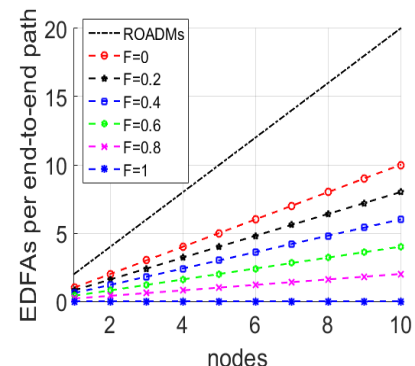

(a)

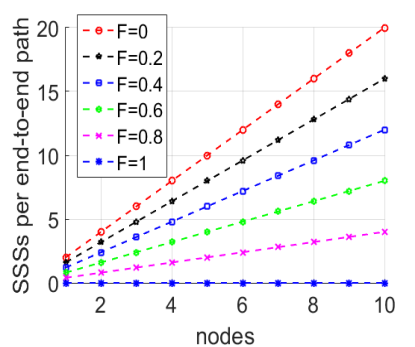

(b)
Fig. 12. (a) Number of EDFAs Used per end-to-end N Path in AoD Nodes. (b) Number of SSSs Used per end-to-end Path in AoD Nodes.

networking scenarios could be further exploited with SBVTs (sliceable bitrate variable transponders) to increase the overall network capacity and efficiency.

\section{E. Network-wide analysis of Fiber Switch Requests}

The proportion of fiber switch requests directly affects the number of EDFAs used in an AoD node. Since the fiber witch request are equally distributed among the nodes in the network, the mean number of EDFAs used in each node is the same. In particular, fewer components are used in the AoD nodes for high proportions of fiber switch requests. Therefore, signals suffer less power loss in the path and less EDFAs are required. In the following study, the system is fed with a $0 \mathrm{dBm}$ flat input power, and the link distance is assumed to be $100 \mathrm{~km}$. From Fig. 12(a) we can see that the slopes of different proportions of fiber switch request increase linearly. Comparing to the traditional ROADMs, which place EDFAs at the input and output ports, the number of EDFA used has reduced $50 \%$ when there is no fiber switch requests and more resource can be saved as the proportion of fiber switch requests increases. Specifically, for $\mathrm{F}=0$, we observe that the EDFAs used in the AoD node increase linearly corresponding to one EDFA per node. Meanwhile, in Fig. 12(b), the average SSS used in the AoD node is 2 SSSs per node per path, which means the two stages of the AoD node are used. On the contrary, when $\mathrm{F}=1$, no EDFAs nor SSSs are used in the AoD nodes. Indeed, a complete fiber switching scenario is required to obtain this outcome because no SSS is required for multiplexing nor demultiplexing. Furthermore, the component loss in this situation is very low, and no EDFAs are used in the AoD node.

Figure 13 shows the normalized OSNR penalty against different fiber switch requests. OSNR penalty when $\mathrm{F}=0$ is used as a reference. We observe that, although the OSNR penalty difference between the scenarios of different fiber switch requests is very small (only 0.035 difference), the maximum OSNR penalty occurs when $\mathrm{F}=1$. We can see as the proportion of fiber switch request increases, the OSNR penalty also increases. This is because in these scenarios, the number of EDFAs and SSSs is small, and the ability of balancing the power is weak comparing to the scenarios with less fiber switch requests. From the obtained results, we can see that, although EDFAs and SSSs bring noise and power loss to the signals, these components can control the power in order to minimize the noise impact on the OSNR performance.

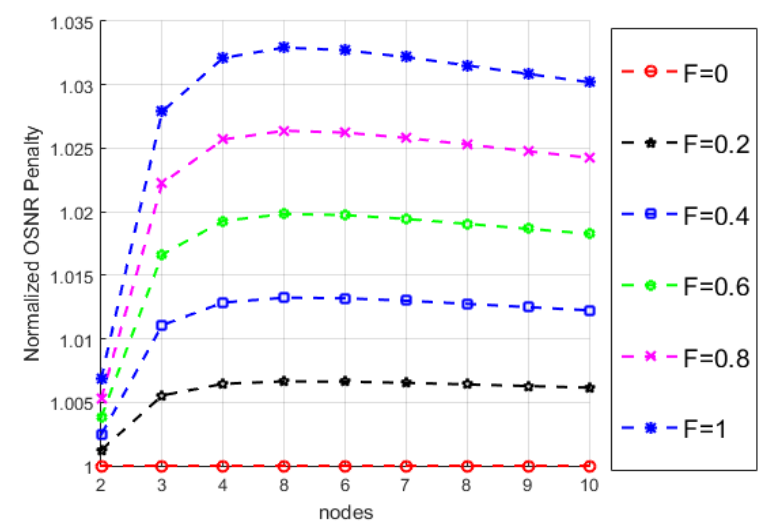

Fig. 13. Normalized OSNR Penalties against Different Fiber Switch Requests and Hops ( $\mathrm{F}=0$ as reference)

\section{F. Network-wide equalization using Power Balance of Fiber switch requests}

In Fig. 14, we present the power difference between the maximum and minimum power levels collected across all the output ports in the AoD nodes. Keep in mind that channels within same output power levels are power balanced (as per previous sections) and this reflects mostly power difference between different output ports. Here we consider a non-flat input power with $20 \mathrm{~dB}$ of difference as input to the network. Figure 14 (a) shows the result for a scenario in which the AoD nodes are connected directly without EDFA chains in-between and Fig.14 (b) reports the result with EDFA chains between AoD nodes. In Fig. 14(b), we observe that the EDFA chain between the adjacent nodes can minimize the power difference among the output ports. In particular, the power difference is reduced from 20 $\mathrm{dB}$ to less than $1 \mathrm{~dB}$ at the output port of the second node. On the contrary, the power difference can still reach up to about $4 \mathrm{~dB}$ after 9 hops when AoD nodes are connected directly without EDFA chain in-between as shown in Fig. 14(b) for $F>0$. Here we further analyze this result observing the combination of power balance and fiber switch requests accounting for the impact on the components available on a composed AoD node. Indeed, note that in Fig. 14(a), the power difference can be reduced to $0 \mathrm{~dB}$ at the output port of the first node when $F=0$. However, as the proportion of fiber switch request increases, the ability to control power decreases. Indeed, when $\mathrm{F}=1$, only fiber switching occurs and no SSSs is used for controlling the power. Therefore, EDFAs are used only when the output power is lower than $-3 \mathrm{dBm}$ creating a high unbalanced power situation. Instead, in Fig. 14(b) we can see that, when

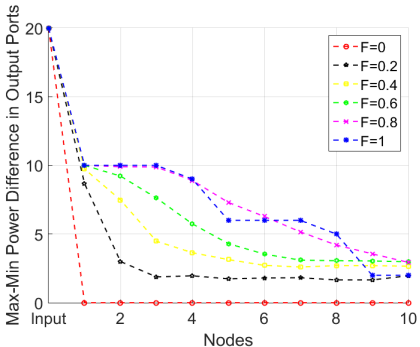

(a)

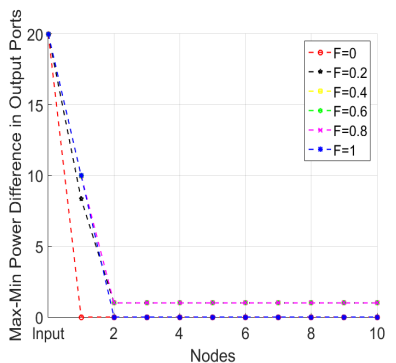

(b)
Fig. 14. Max-Min Power Difference in Output ports

(a) No EDFA Chain between AoD nodes

(b) EDFA Chain between AoD nodes 
$\mathrm{F}=0$ and $\mathrm{F}=1$, the max-min power difference remains $0 \mathrm{~dB}$. This is because in this two scenarios, all the paths travel always across the same architecture (two-stage SSS when $\mathrm{F}=0$ or one optical cross-connection when $\mathrm{F}=1$ ). In other scenarios, the power difference among the output ports is $1 \mathrm{~dB}$.

\section{CONCLUSION}

This work investigates network function programmability for open and disaggregated nodes able to support optical functions virtualization. We presented ONetFuS, a novel Optical Network-wide Function Synthesis algorithm, which combines a Switching Synthesis and Placement Algorithm (SSPA) and an OSNR and Power Balance aware EDFA Placement (OPB-EDFA-Placement) algorithm. ONetFuS computes different AoD instances in network scenarios including wavelength, waveband and fiber switch requests.

Our results show that the architectures built by our proposed SSPA notably reduce the use of optical crossconnections in AoD nodes. Secondly, we studied the OPBEDFA-Placement algorithm performance in network scenarios analyzing OSNR variation and OSNR penalty against different network design parameters. Our results showed that the OSNR variation is mainly introduced by the variation of the input power level. Additionally, we observed that the input power and the distance between adjacent nodes significantly affect the OSNR performance. However, the difference in OSNR penalty decreases as the number of hops increases. Thirdly, we compared the number of EDFAs used in the network with different proportion of fiber switch requests and its impact on OSNR against the EDFAs used in a traditional ROADM network. The results showed that the proportion of fiber switch requests affects the number of EDFAs and SSSs used in the AoD nodes. In addition, the number of EDFAs used in the AoD node can be reduced by $50 \%$ when compared to traditional ROADM architectures. Finally, we verified that EDFAs and SSSs are the key components for OSNR awareness and power balancing. These components introduce noise and power loss to the network. However, with proper placement and configuration of their optical functionalities, power balancing, optical cross connections and OSNR performance can be optimized.

\section{ACKNOWLEDGMENT}

This work is partially supported by EPSRC grants EP/L0207070/1 SONATAS and H2020 dRedBox project with grant agreement 687632. The authors also thank the financial support of FUNTTEL under the project 100GETH, and CNPq (grant 312047/2015-0).

\section{REFERENCES}

[1] J. Berthold, A. A. M. Saleh, L. Blair, and J. M. Simmons, "Optical networking: Past, present, and future," Journal of Lightwave Technology, vol. 26, no. 9, pp. 1104-1118, May 2008.

[2] O. Gerstel, M. Jinno, A. Lord, and S. B. Yoo, "Elastic optical networking: A new dawn for the optical layer?," IEEE Communications Magazine, vol. 50, n. 2, pp. s12-s20, 2012.

[3] J. Fernandez-Palacios, V. López, B. Cruz, and O. G. de Dios, "Elastic optical networking: An operators perspective,"
European Conference on Optical Communication, Cannes, France, 2014.

[4] J. L. Vizcaíno, Y. Ye, V. López, F. Jiménez, R. Duque, and P. M. Krummrich, "Cost evaluation for flexible-grid optical networks," Globecom Workshops, pp. 358-363, Anaheim, California, USA, December 2012.

[5] X. Yu, M. Tornatore, M. Xia, Y. Zhao, J. Zhang, and B. Mukherjee, "Brown-field migration from fixed grid to flexible grid in optical networks," Optical Fiber Communication Conference, pp. W1I-4, Los Angeles, California, USA, 2015.

[6] T. Rahman, A. Napoli, D. Rafique, B. Spinnler, M. Kuschnerov, I. Lobato, B. Clouet, M. Bohn, C. Okonkwo, and H. Waardt, "On the Mitigation of Optical Filtering Penalties Originating From ROADM Cascade," Photonics Technology Letters, vol. 26, no. 2, pp. 154-157, 2014.

[7] R. Younce, J. Larikova, and Y. Wang, "Engineering 400G for colorless-directionless-contentionless architecture in metro/regional networks [invited]," Journal of Optical Communications and Networking, vol. 5, no. 10, pp. A267A273, 2013.

[8] The Open ROADM Multi-Source Agreement, available at: http://www.openroadm.org/

[9] N. Amaya, G. Zervas, and D. Simeonidou, "Architecture on demand for transparent optical networks," International Conference on Transparent Optical Networks (ICTON), Stockholm, Sweden, 2011.

[10] N. Amaya, G. Zervas, and D. Simeonidou, "Introducing node architecture flexibility for elastic optical networks," Journal of Optical Communications and Networking, vol. 5, no.6, pp. 593608, 2013.

[11] M. Garrich, N. Amaya, G. Zervas, P. Giaccone, and D. Simeonidou, "Architecture on Demand: Synthesis and scalability," Optical Network Design and Modeling (ONDM), Colchester, U.K., 2012.

[12] M. Dzanko, B. Mikac, N. Amaya, G. Zervas, and D. Simeonidou, "Availability analysis of optical cross-connect implemented by architecture on demand," International Conference Transparent Optical Networks, Coventry, U.K., 2012.

[13] A. Muhammad, G. Zervas, N. Amaya, D. Simeonidou and R. Forchheimer, "Introducing flexible and synthetic optical networking: planning and operation based on network function programmable ROADMs," Journal of Optical Communications and Networking, vol. 6, no. 7, pp.635-648, July 2014.

[14] "Optical Layer Pluggables: The End Of Hard Choices In The Metro?: Examining Three Optical Layer Architectures", Coriant Whitepaper, available at: http://www.coriant.com/misc/downloads/Coriant_WP_Optical_ Layer_Pluggables.pdf

[15] M. Garrich, N. Amaya, G. S. Zervas, J. Oliveira, P. Giaccone, A. Bianco, D. Simeonidou, and J. Oliveira, "Architecture on demand design for high-capacity optical SDM/TDM/FDM switching," Journal of Optical Communications and Networking, vol. 7, no. 1, pp. 21-35, 2015.

[16] M. Džanko, M. Furdek, G. Zervas, and D. Simeonidou, "Evaluating availability of optical networks based on selfhealing network function programmable ROADMs," Journal of Optical Communications and Networking, vol.6, no. 11, pp. 974-987, 2014.

[17] S. Fujii, Y. Hirota, T. Watanabe and H. Tode, "Dynamic spectrum and core allocation with spectrum region reducing costs of building modules in AoD nodes," Telecommunications Network Strategy and Planning Symposium (Networks), Funchal, Portugal, 2014.

[18] H. Yuan, M. Garrich, E. Hugues Salas, G. S. Zervas, and D. E. Simeonidou, "Function Placement and Configuration for Power Balanced Network Function Programmable Optical Nodes," in 
Optical Fiber Communication Conference, Los Angeles, California, USA, p. W3J.2, 2015.

[19] C. Diniz, M. Garrich, G. Zervas, and D. Mello, "OSNR-aware Control of Optical White Boxes on Elastic Optical Networks," accepted at Asia Communications and Photonics Conference, Wuhan, China, November 2016.

[20] V. López, O. G. de Dios, L. Contreras, J. Foster, H. Silva, L. Blair, J. Marsella, T. Szyrkowiec, A. Autenrieth, C. Liou, A. Sasdasivarao, S. Syed, J. Sun, B. Rao, F. Zhang, and J. Fernández-Palacios, "Demonstration of SDN orchestration in optical multi-vendor scenarios," Optical Fiber Communication Conference, Los Angeles, California, USA, March 2015.

[21] M. Cantono, R. Gaudino, and V. Curri, "Potentialities and Criticalities of Flexible-Rate Transponders in DWDM Networks: A Statistical Approach," Journal of Optical Communications and Networking, vol. 8, no. 7, pp. A76-A85, 2016.

[22] YANG - IETF NETCONF Data Modeling Language Working Group, Oct. 2010. Available: https://tools.ietf.org/html/rfc6020

[23] M. Dallaglio, N. Sambo, J. Akhtar, F. Cugini, and P. Castoldi, "YANG Model and NETCONF Protocol for Control and Management of Elastic Optical Networks," Optical Fiber Communication Conference, Anaheim, California, USA, 2016.

[24] Jobaida Akhtar, "YANG modeling of network elements for the management and monitoring of Elastic Optical Networks," IEEE International Conference on Telecommunications and Photonics, Dhaka Dhaka, Bangladesh, 2015.

[25] M. Dallaglio, N. Sambo, F. Cugini, and P. Castoldi, "Management of sliceable transponder with NETCONF and YANG," Optical Network Design and Modeling, Cartagena, Spain, 2016.

[26] M. Garrich, N. Amaya, G. Zervas, P. Giaccone, and D. Simeonidou, "Power consumption analysis of architecture on demand," European Conf. on Optical Communication, Amsterdam, Netherlands, 2012.

[27] A. Muhammad, M. Furdek, P. Monti, L. Wosinska, and R. Forchheimer. "Dynamic provisioning utilizing redundant modules in elastic optical networks based on architecture on demand nodes," European Conference on Optical Communication, Cannes, France, September 2014.

[28] M. Furdek, M. Dzanko, M. Matanic, I. Boric, L. Wosinska, and B. Mikac. "Multi-hour network provisioning utilizing function programmable ROADMs," International Conference on Transparent Optical Networks, Budapest, Hungary, 2015.

[29] A. Ahmad, A. Bianco, E. Bonetto, M. Garrich, J. Oliveira. "Switching node architectures in flexible-grid networks: A performance comparison," Optical Network Design and Modeling, Stockholm, Sweden, May 2014.

[30] Finisar's WaveShaper. Available at: http://www.finisar.com/.

[31] E. Desurvure, Erbium-Doped Fiber Amplifiers Principles and Applications. New York: John Wiley \& Sons, 1994. 